\title{
Optimization of Multistage Depressed Collectors
}

\author{
Tushar K. Ghosh, Member, IEEE, and Richard G. Carter, Senior Member, IEEE
}

\begin{abstract}
Random walk and genetic algorithm techniques have been implemented in a 3-D collector simulation code to automate the design optimization of multistage depressed collectors. An axisymmetric four-stage collector and an asymmetric two-stage collector have been optimized following both approaches. Procedures for the implementation of these methods in any suitable code and simulated performance of the collectors are demonstrated. The results show significant improvement in the collector performances due to optimization. A comparison between the optimum collector performances that were obtained using these methods has also been carried out.
\end{abstract}

Index Terms-Genetic algorithms, multistage collector, optimization methods, random walk.

\section{INTRODUCTION}

$\mathbf{T}$ HE MULTISTAGE collector is a critical component of vacuum microwave and millimeter-wave devices where efficiency is an important parameter, such as traveling-wave tube amplifiers in spaceborne and airborne applications, gyrotrons in plasma applications, and klystrons and inductive output tubes in television transmissions. Therefore, optimization of multistage collector performance is essential to maximize recovered power from spent electron beam. Since the inception of modern multistage collectors, different techniques have been adopted to achieve maximum collector and overall efficiencies [1]-[4]. Secondary electrons play a determining role in collector performance. Fabrication of electrodes with materials of low secondary electron emission coefficient reduces the effect of secondaries [5]-[8]. A transverse magnetic field may be applied in the collector region to reduce backstreaming of reflected primary and secondary electrons. Sometimes, introduction of asymmetry in the geometry may be useful to recapture the secondary electrons [9], [10]. Another way of improving multistage collector performance is shaping of the electric and magnetic fields inside the collector in such a way that the electrons land on the electrodes perpendicularly. This reduces their transverse energy and, hence, loss in the form of heat. However, little has been published on methods of choosing the geometry of the collector electrodes to optimize the collector performance. It is not very efficient to optimize the collector performance manually as many geometrical parameters, e.g., angle, inner diameter, outer diameter, and thickness of each

Manuscript received February 21, 2007; revised May 2, 2007. The work of T. K. Ghosh was supported in part by the Association of Commonwealth Universities, U.K., in the form of a Commonwealth Scholarship in the Ph.D. program and in part by the IEEE Electron Devices Society in the form of an IEEE-EDS Graduate Student Fellowship Award in 2001. The review of this paper was arranged by Editor W. Menninger.

T. K. Ghosh is with the Helix Engineering, e2v technologies, Chelmsford CM1 2QU, U.K. (e-mail: tushar.ghosh@e2v.com).

R. G. Carter, is with the Department of Engineering, Lancaster University, Lancaster LA1 4YR, U.K. (e-mail: r.carter@lancaster.ac.uk).

Digital Object Identifier 10.1109/TED.2007.900003 electrode and the spacing between the electrodes, need to be considered. Instead, a search algorithm can be used to find the collector geometry for optimum performance. Different techniques such as random walk, genetic algorithm, and simulated annealing have been tried in the past to automate the design process. The simulated annealing technique was implemented in two dimensions to optimize the geometry of an axisymmetric collector, but the feasibility of its fabrication was not considered [11]. Optimization of multistage collector geometry using random walk and genetic algorithm was first demonstrated by the authors [12], [13]. The implementation of these techniques in a 3-D collector simulation code and the simulated performance of optimized axisymmetric and asymmetric collectors are presented here. Any electrostatic code with the capability of simulating the electron trajectories can be used as a basis for implementing these techniques for automated optimization.

\section{Development of 3-D PACKAGE}

The mainframe version of Lancaster KOBRA, ${ }^{1}$ which was developed earlier in a European Space Research and Technology Centre sponsored project [14], was used as a basis for the implementation of both random walk and genetic algorithms. Due to the automated nature of the algorithm, it was necessary to make some changes in the package as well as in its preprocessor and postprocessor [13].

The option to input the geometry, the mesh information, the electrode potentials, and the boundary conditions through a data file has been created, which helps in changing the shape and size of any electrode easily due to parameterization. To generate the starting conditions of the electron trajectories, the parameters of the spent electron beam, i.e., position and velocity components, have been generated using a large-signal model. An interface with a 2.5-D model SUNRAY-3D [15] has been introduced to convert the initial conditions of the electron trajectories into a 3-D format.

To implement the optimization algorithms, it is necessary to combine the geometry generator, the Poisson solver, the trajectory solver, the preprocessor, the postprocessor, and the optimization codes. The present version of the package runs in the UNIX operating system in a batch mode, and it terminates once the user-specified iteration number is reached or the target collector efficiency is achieved. For each iteration, the simulation time for any collector geometry with 750000 nodes was nearly 15 min in a $2.8-\mathrm{GHz}$ personal computer with a 2-GB random access memory. After each cycle, the collector geometry is stored in a parameterized form along with its analysis report. This makes it possible to analyze the history of the

${ }^{1}$ KOBRA is distributed by INP-DME, 65205 Wiesbaden, Germany. 


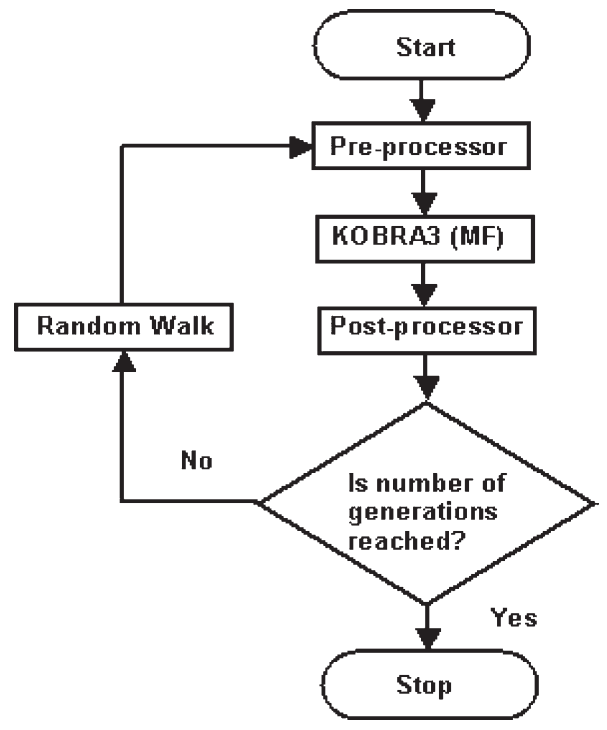

Fig. 1. Flow diagram of the package incorporating random walk algorithm.

changes being made in the collector geometry and their impact on its performance during the optimization process. The collector geometry corresponding to the best efficiency achieved so far is stored in a separate file, which can be retrieved in case of intentional or accidental stopping during execution.

\section{Optimization Using RAndom Walk Method}

The simplest random walk algorithm starts from a point, follows a random path, and looks for the object function values at different points in a finite search space considering only one point at a time. Several variations of the algorithm are possible depending on the application to a specific problem [16], [17]. This algorithm is most effective in cases where the search space and the number of possibilities are small.

\section{A. How It Works}

A basic flowchart for the design optimization package using random walk approach is shown in Fig. 1. The preprocessor uses the geometry and trajectory data to generate a suitable input file for the simulator. The postprocessor computes the efficiency of the collector, which acts as the object function value. If after any iteration the performance is better than the best value achieved so far, then the search operation starts again from this point; otherwise, it starts from the previous best geometry. The decision to choose the point of the next computation is made by the random walk algorithm. After several iterations, if no improvement in collector performance is observed, the user may start the optimization process again from the initial starting point along a different path. Otherwise, the whole process will continue until the number of iterations specified by the user.

A clear advantage of this technique over enumerative methods is that all dimensions of the collector electrodes are changed simultaneously. The detailed flowchart of the random walk section is shown in Fig. 2.

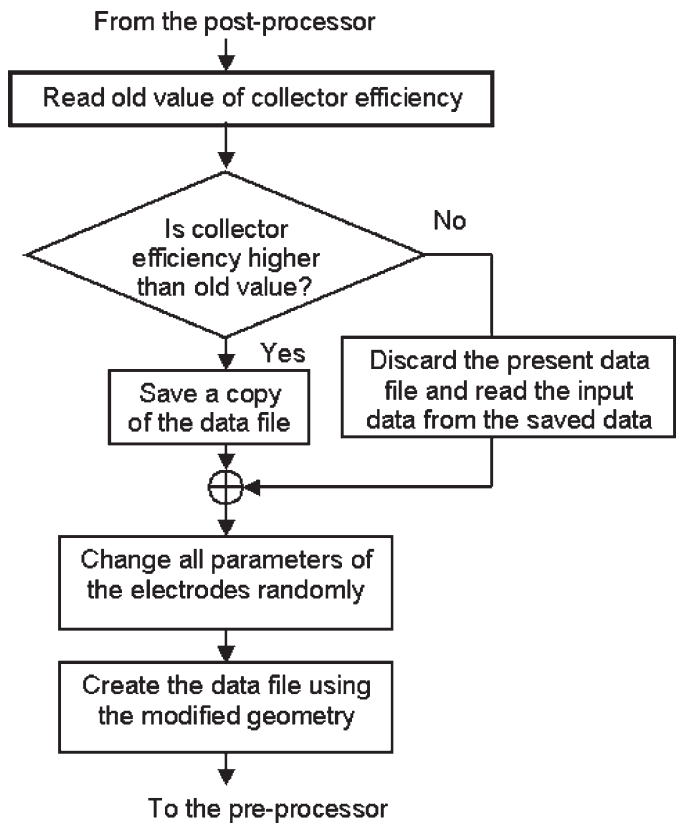

Fig. 2. Detailed flowchart for the random walk section.

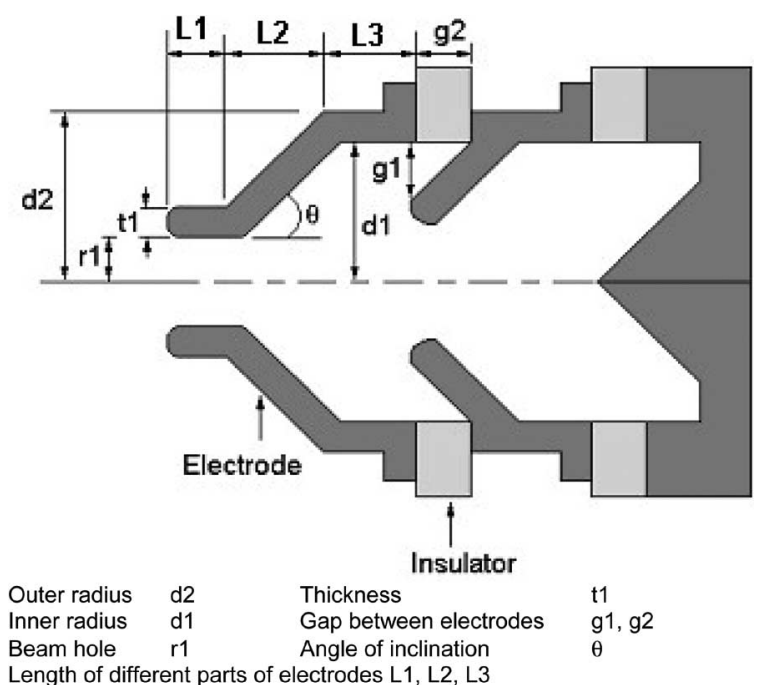

Fig. 3. Different dimensions of an electrode of a three-stage collector.

\section{B. How to Create a New Geometry}

New collector geometries are created by randomly changing the electrode dimensions (e.g., length, inner diameter, outer diameter, and angle of inclination with the axis) in the intermediate parameterized data file. The maximum and minimum limits of the parameter to be changed (increment or decrement) are based on the sensitivity of any dimension and the feasibility of its fabrication. However, changes should be made in very small steps to ensure that the changes in the collector efficiency due to changes in the electrode dimensions are small. In general, the most sensitive dimension is changed least. A pseudorandom number generator produces a number between 0 and 1 for each electrode dimension to be decreased or increased, respectively.

The dimensions of the electrodes of a three-stage axisymmetric collector, which are commonly changed to create a new geometry, are shown in Fig. 3. These parameters may vary 
depending on the shape of an electrode. The complexity of the problem can be understood from the number of dimensions to be changed for a four-stage or a five-stage collector.

Precautions are taken while changing the dimensions of the electrodes.

1) Gap between the electrodes: In multistage collectors, it is common to have an electrode that lies inside the previous electrode (in the direction of the interaction circuit). A minimum value for $\mathrm{g} 2$ ensures a sufficient gap between the high-voltage electrodes to avoid sparking, and a maximum limit on the gap prevents the electrons from hitting the high-voltage insulators.

2) Outer radius: The maximum value of the outer radius depends on the inner radius and electrode thickness.

3) Inner radius: In the case of a cylindrical electrode, the inner radius (d1) should be large enough to maintain a reasonable gap (g1) between the electrodes.

4) Beam hole radius: This dimension should be higher than the beam radius to accommodate the expanded beam. The spent beam electrons must not be allowed to strike the front face of the electrode (surface toward the interaction circuit). Otherwise, the secondaries and the reflected primaries generated here see an accelerating field, which causes them to stream back toward the interaction circuit.

5) Thickness of the electrodes: The minimum electrode thickness should be fixed at a practical value.

6) Angle of inclination: The minimum and maximum are set at some practical values.

Other than the aforementioned parameters, several other parameters, such as the length of different parts of an electrode and the angles of the two end planes of the electrodes, may also be changed. The change in the angles of the planes is necessary for the optimization of asymmetric tilted electric field (TEF) collectors. However, for symmetric collectors, these angles are always kept fixed at $90^{\circ}$ to the axis.

\section{Results and Validation of the Package IMPLEMENTING RANDOM WALK ALGORITHM}

The random walk algorithm has been tested by optimizing the design of a four-stage axisymmetric collector and a twostage asymmetric collector. After several trials, it was observed that the present package was capable of achieving a significant improvement in the efficiency for both the collectors.

\section{A. Axisymmetric Collector}

In Table I, the efficiencies of a four-stage axisymmetric collector for different trials are shown, where the initial collector geometry is the same in all cases. Only the results of the best four trials are shown in the table. For the initial geometry shown in Fig. 4, the collector efficiency is $70 \%$, considering only primary electrons. The best collector efficiency in each case is shown after every 50 iterations. The amount of change in the dimensions of the collector electrodes is random in any trial in each run. For this reason, the intermediate steps are different, and the difference between the optimized efficiencies for all four cases is evident. The best efficiency among all these trials
TABLE I

OPTIMIZED COLLECTOR EFFICIENCIES FOR DIFFERENT TRIALS (FOUR-STAGE AXISYMMETRIC COLLECTOR)

\begin{tabular}{|c|c|c|c|c|}
\hline After & Trial 1 & Trial 2 & Trial 3 & Trial 4 \\
\hline Iteration & $\begin{array}{c}\text { Efficiency } \\
\text { (\%) }\end{array}$ & $\begin{array}{c}\text { Efficiency } \\
\text { (\%) }\end{array}$ & $\begin{array}{c}\text { Efficiency } \\
\text { (\%) }\end{array}$ & $\begin{array}{c}\text { Efficiency } \\
\text { (\%) }\end{array}$ \\
\hline 50 & 85.94 & 83.41 & 83.11 & 83.57 \\
\hline 100 & 87.45 & 83.45 & 83.11 & 83.57 \\
\hline 150 & 87.45 & 83.46 & 83.11 & 83.57 \\
\hline 200 & 87.45 & 83.77 & 83.90 & 84.30 \\
\hline 250 & 87.45 & 84.88 & 87.37 & 84.30 \\
\hline 300 & 87.45 & 84.88 & 88.97 & 85.52 \\
\hline 350 & 87.45 & 84.88 & 89.20 & 85.52 \\
\hline 400 & 87.45 & 84.88 & 89.20 & 86.24 \\
\hline 450 & 87.45 & 84.91 & 89.20 & 86.24 \\
\hline 500 & 87.45 & 84.91 & 89.20 & 86.24 \\
\hline
\end{tabular}

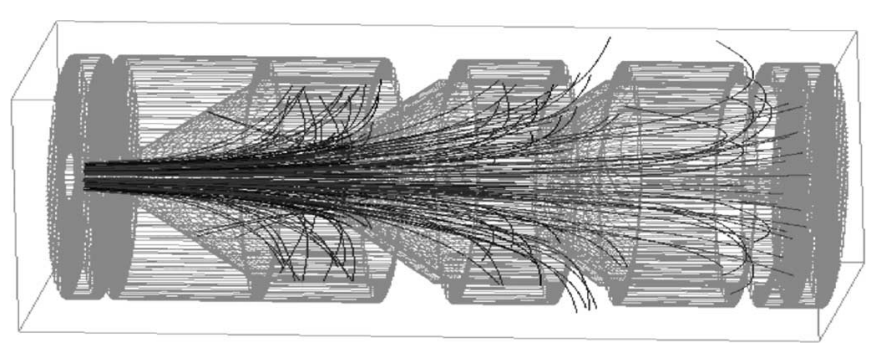

Fig. 4. Initial geometry of the four-stage collector with $70 \%$ efficiency.

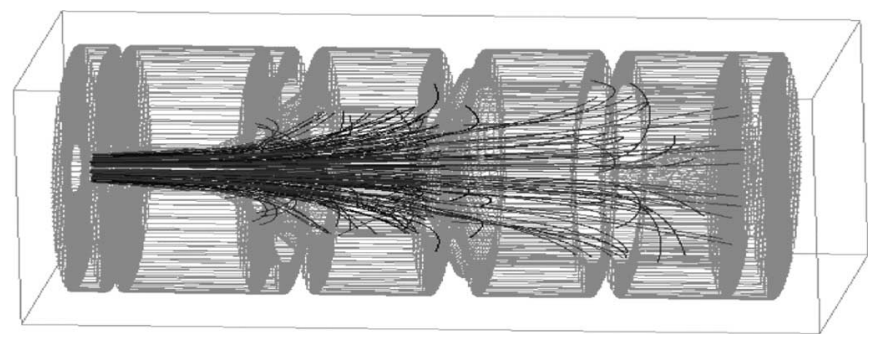

Fig. 5. Final geometry of the four-stage collector with $89.2 \%$ efficiency.

is $89.2 \%$. The improvement in the collector efficiency is more than 19 percentage points for the optimized geometry shown in Fig. 5. A careful look at the initial geometry shows that some of the trajectories are coming out through the gap between the electrodes and may therefore hit the high-voltage insulators and damage them. However, due to the optimization of the gap length between the electrodes, this phenomenon is missing in the optimized collector. If the number of trials is increased, then the efficiency may be increased further by a small amount.

\section{B. Asymmetric Collector}

A two-stage asymmetric TEF collector has been optimized using the same algorithm. The collector efficiency for the initial geometry is $79 \%$, considering only primary electrons. Table II shows the computed efficiency after every 50 iterations for the best four trials. For this collector, the optimized efficiency is better than $82 \%$. It should be observed here that no improvement in collector efficiency was achieved after 200 iterations. The asymmetric two-stage collectors before and after optimization are shown in Figs. 6 and 7, respectively. The improvement in efficiency is better than 3 percentage points. 
TABLE II

OPTIMIZED COLLECTOR EFFICIENCIES FOR DIFFERENT TRIALS (TWO-STAGE TEF COLLECTOR)

\begin{tabular}{|c|c|c|c|c|}
\hline After & Trial 1 & Trial 2 & Trial 3 & Trial 4 \\
\hline Iteration & $\begin{array}{c}\text { Efficiency } \\
\text { (\%) }\end{array}$ & $\begin{array}{c}\text { Efficiency } \\
\text { (\%) }\end{array}$ & $\begin{array}{c}\text { Efficiency } \\
\text { (\%) }\end{array}$ & $\begin{array}{c}\text { Efficiency } \\
\text { (\%) }\end{array}$ \\
\hline 50 & 82.05 & 82.42 & 81.50 & 81.87 \\
\hline 100 & 82.05 & 82.42 & 81.87 & 81.87 \\
\hline 150 & 82.05 & 82.42 & 81.87 & 82.05 \\
\hline 200 & 82.05 & 82.42 & 81.87 & 82.24 \\
\hline 250 & 82.05 & 82.42 & 81.87 & 82.24 \\
\hline 300 & 82.06 & 82.42 & 81.87 & 82.24 \\
\hline 350 & 82.06 & 82.42 & 81.87 & 82.24 \\
\hline 400 & 82.06 & 82.42 & 81.87 & 82.24 \\
\hline 450 & 82.06 & 82.42 & 81.87 & 82.24 \\
\hline 500 & 82.06 & 82.42 & 81.87 & 82.24 \\
\hline
\end{tabular}

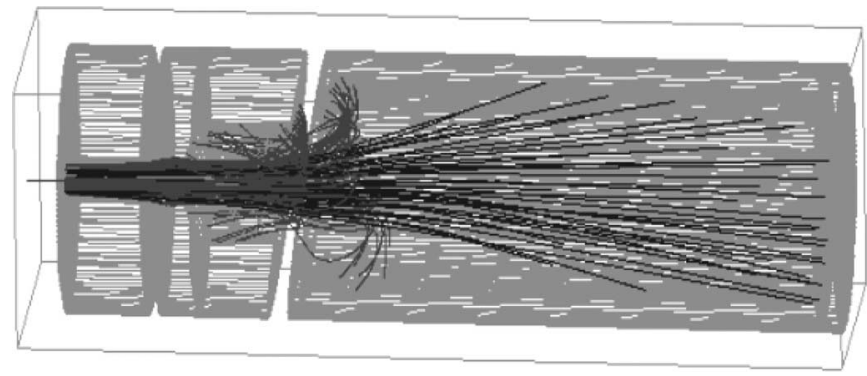

Fig. 6. Initial geometry of the two-stage TEF collector with $79 \%$ efficiency.

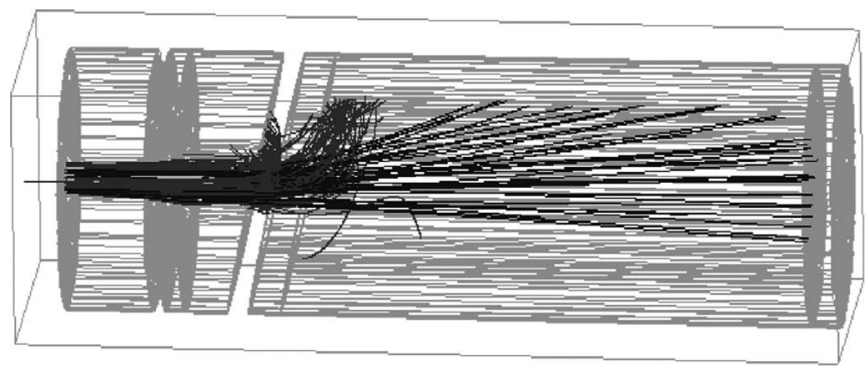

Fig. 7. Final geometry of the two-stage TEF collector with $82 \%$ efficiency.

\section{Optimization Using a Genetic Algorithm}

The random walk method has major limitations, such as the possibility of being trapped at a local maximum and the requirement of a large number of trials to find the optimized geometry. These can be overcome by a more efficient technique, namely the genetic algorithm [18]-[21]. This method is based on natural phenomena and the theory of the survival of the fittest. It generates a new set of data through natural selection and genetics through random choice.

\section{A. Implementation of the Genetic Algorithm}

A basic flowchart for the automated optimization process implementing the genetic algorithm is similar to Fig. 1 except that the genetic algorithm replaces the random walk algorithm. However, this method is more complicated than the random walk approach, and it is also more difficult to implement.

The detailed flow diagram of the genetic algorithm process is shown in Fig. 8. The sequence of events implementing the ge- netic algorithm is described in six steps for better understanding of the procedure.

1) From previous experience or by scaling an existing collector, a new geometry is created as a preliminary design.

2) Using a nonrepetitive random number generator, a population of chromosomes (a string of binary digits) of equal length is created in such a way that no two members in the population are the same. A brief description on how to choose the size of the population and the length of the chromosome is given in the next section.

3) A set of collectors is produced using the chromosomes and the basic geometry. For each collector, the simulator is run until a converged solution is achieved.

4) The efficiency of each collector, which is calculated using the postprocessor, is then used as the object function, or fitness value, for the corresponding chromosome. A new population of collectors is generated from the existing population through the three main operators: 1) reproduction; 2) crossover; and 3) mutation. These three operators are discussed in detail in a later section.

5) The function of the genetic algorithm for this generation is complete, and the process is repeated for the next generation of collectors. The best collector geometry, corresponding to the best efficiency achieved in each generation, is used as the basic geometry for the next generation.

6) Steps 3-5 are repeated until the number of generations (specified by the user) is reached. If in any generation the best efficiency is not better than the previous best, then the total population is discarded, and a new population is created from the previous population.

\section{B. How to Create a New Geometry}

To introduce diversity in the optimization procedure, the search is started from a large population; i.e., a large number of geometries are created that covers many points in the search space for a possible solution. This also provides a large number of alternative paths toward optimization.

With a chromosome length of 10 , there are $2^{10}=$ 1024 points in the search space. The larger the length of the chromosome, the larger the number of points it covers in the search space. In the present problem, each binary bit of a chromosome corresponds to a simple electrode, which makes the chromosome length same as the number of simple electrodes. It is worth mentioning here that in the present simulation package, the number of electrodes is not the same as the number of collector stages as the complex electrode geometry is created by combining predefined simple electrodes, as shown in Fig. 9. It is also possible to associate each binary bit with a dimension of an electrode. In that case, the chromosome length will be large.

To create a new collector from a basic geometry, the dimensions of an electrode (e.g. length, angle, inner diameter, and outer diameter) are increased if the bit is 1 and decreased if the bit is a 0 . The changes in the dimensions follow the same logic for the random walk algorithm. Thus, a new population of collectors corresponding to each member chromosome is formed. 


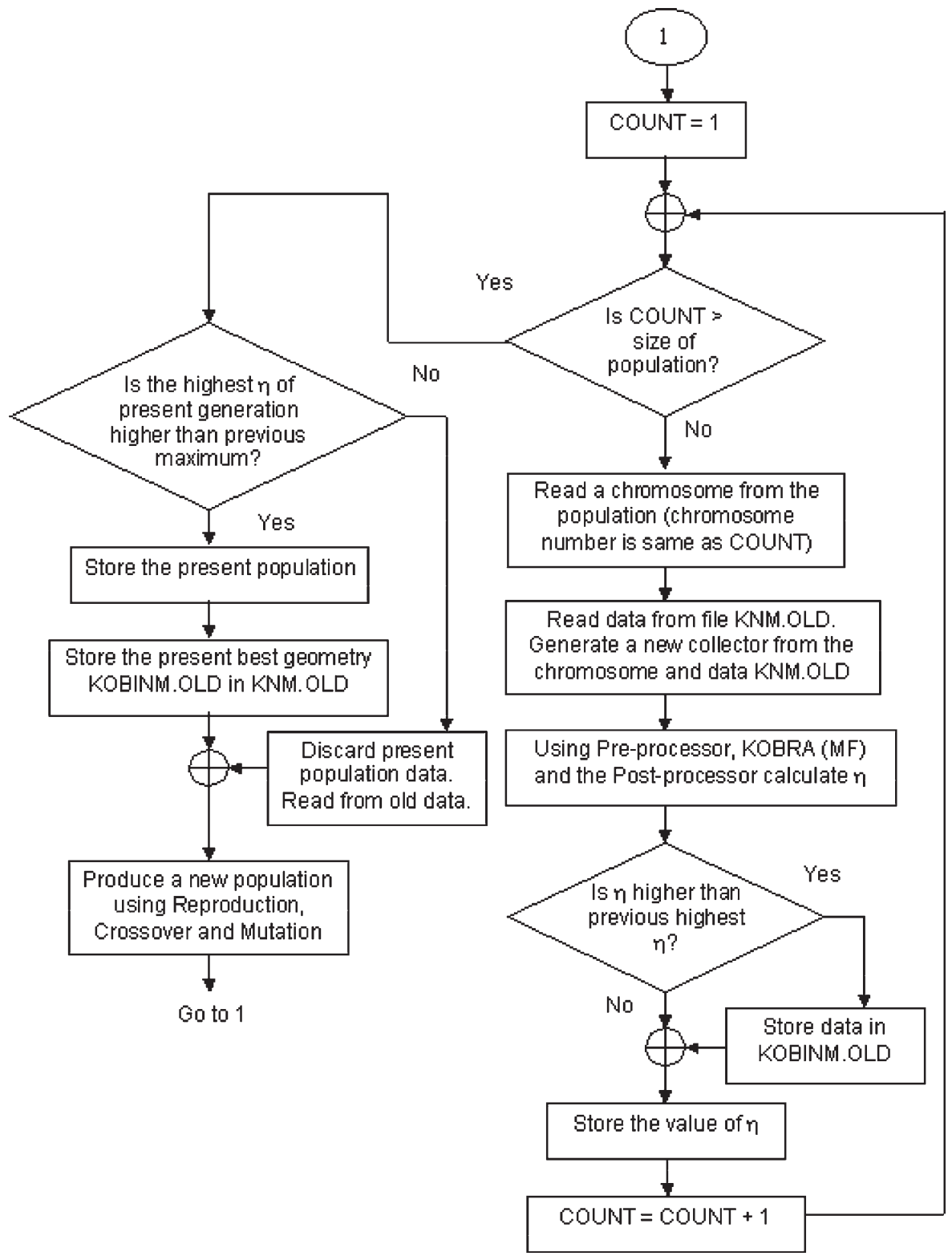

Fig. 8. Flow diagram of the genetic algorithm section. $\eta$ denotes the collector efficiency; KNM.OLD stores the best collector geometry data achieved until that generation; KOBINM.OLD stores the best geometry data of the collector in any particular generation, and it is generated by the algorithm using the chromosome and the data file KNM.OLD.

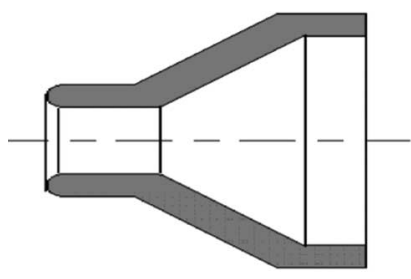

(a)

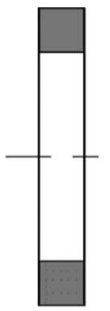

(b)

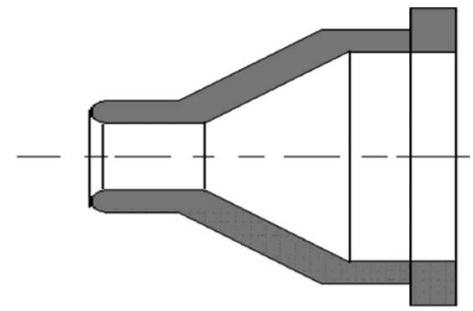

(c)

Fig. 9. Electrode shapes. (a) Funnel. (b) Cylinder. (c) Complex shape formed by combining funnel and cylinder.

\section{Reproduction, Crossover, and Mutation}

An example of the three basic operators (reproduction, crossover, and mutation) to create a new population is presented here. A typical randomly generated population is shown in the second column of Table III, where the size of the population is 4 and the length of each chromosome is 10 . A new collector geometry corresponding to each chromosome is produced from the basic collector shape. The computed efficiency of each collector is shown in the third column. 
TABLE III

TYPICAL REPRODUCTION PROCESS

\begin{tabular}{cccc}
$\begin{array}{c}\text { String } \\
\text { No. }\end{array}$ & $\begin{array}{c}\text { Random Initial } \\
\text { Population }\end{array}$ & $\begin{array}{c}\text { Collector } \\
\text { Efficiency (\%) }\end{array}$ & $\begin{array}{c}\text { Number of } \\
\text { Offspring }\end{array}$ \\
\hline 1 & 1010110111 & 75 & 2 \\
2 & 0101001100 & 45 & 1 \\
3 & 0011010110 & 40 & 1 \\
4 & 0001110001 & 20 & 0 \\
\hline
\end{tabular}

TABLE IV

AFTER REPRODUCTION

\begin{tabular}{cccc}
\hline $\begin{array}{c}\text { String } \\
\text { No. }\end{array}$ & $\begin{array}{c}\text { Population After } \\
\text { Reproduction }\end{array}$ & $\begin{array}{c}\text { String } \\
\text { No. }\end{array}$ & $\begin{array}{c}\text { Randomly Selected } \\
\text { Pairs }\end{array}$ \\
\hline 1 & 1010110111 & 1 & 1010110111 \\
2 & 1010110111 & 3 & 0101001100 \\
3 & 0101001100 & 2 & 1010110111 \\
4 & 0011010110 & 4 & 0011010110 \\
\hline
\end{tabular}

In the reproduction process, the number of offspring of a collector is produced with a probability proportional to its efficiency. The number of offspring generated corresponding to each collector is shown in the fourth column. In the first line, the number of offspring is two as the collector with the best efficiency produces the most offspring, and in the second and third lines, the number of offspring is one each, whereas the member of the population with the worst efficiency dies off. Therefore, in Table IV, the first two chromosomes are the same as the first chromosome in Table III. Similarly, the second and third chromosomes in Table III remain the same before and after reproduction. They become the third and fourth chromosomes, respectively, in Table IV. The first and second columns of this table show the new string numbers and member chromosomes, respectively. These members are then entered into the mating pool where the pair of parents is chosen randomly from the population. In this case, the first and third chromosomes make a pair, and the second and fourth chromosomes make the second pair. The new string number and the chromosomes are shown in the third and fourth columns, respectively. It is worth mentioning that the size of the population is made a constant even number deliberately to keep the population size constant throughout the computation. In the natural world, the population size changes, which is a complex phenomenon and difficult to implement in an optimization using a genetic algorithm. Moreover, an increase in the population number after each iteration will create a huge population after only a few iterations. This will increase the computation time for each trial many fold.

The next process is crossover. In this problem, we have used a single-point crossover, and the probability of crossover is always 1. A random number between " 1 " and "chromosome length -1 " is used as the point of crossover. All digits between "1" and "point of crossover +1 " are swapped between the pair of parents to produce two children. The crossover process is shown in Table V. In the first case, the crossover point is 6. Therefore, the first 7 bits of "Parent 1" chromosome are swapped with the corresponding bits of "Parent 2" chromosome to produce "Child 1" and "Child 2" chromosomes, respectively. In a similar way, other child chromosomes are generated. These children form the new population. The old and new populations are shown in Table VI.
TABLE V

CROSSOVER

\begin{tabular}{cccc}
\hline Parent 1 & 1010110111 & Child 1 & 0101001111 \\
Parent 2 & 0101001100 & Child 2 & 1010110100 \\
& Crossover at 6 (Randomly Chosen) \\
Parent 3 & 1010110111 & Child 3 & 0011110111 \\
Parent 4 & 0011010110 & Child 4 & 1010010110 \\
\multicolumn{4}{c}{ Crossover at 3 (Randomly Chosen) } \\
\hline
\end{tabular}

TABLE VI

Old And New Populations (Before ANd After Crossover)

\begin{tabular}{ccc}
\hline String No. & Old Population & New Population \\
\hline 1 & 1010110111 & 0101001111 \\
2 & 1010110111 & 1010110100 \\
3 & 0101001100 & 0011110111 \\
4 & 0011010110 & 1010010110 \\
\hline
\end{tabular}

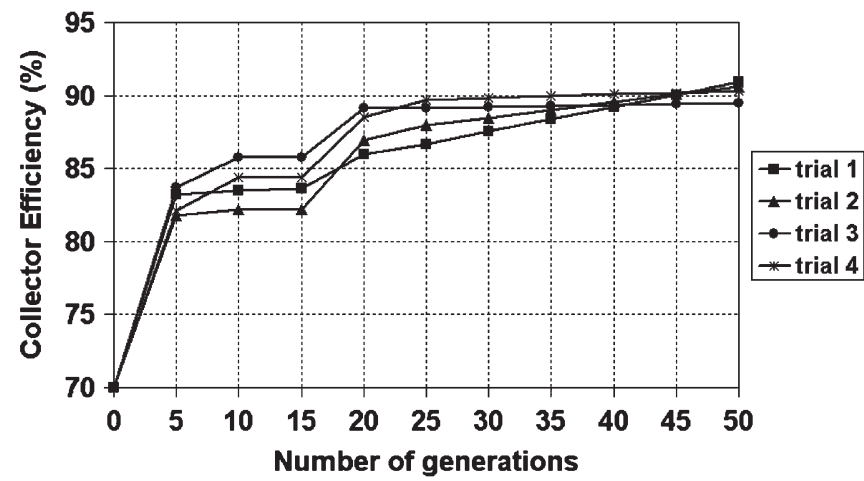

Fig. 10. Improvement in efficiency of a four-stage axisymmetric collector versus the number of generations (the starting geometry is the same for all trials).

Mutation is carried out when all the chromosomes in a population are the same. A random number is generated between " 1 " and "chromosome length" that determines the position of the bit where mutation is to be done. This position is different for each chromosome.

\section{Results AND VALIDATION OF THE PACKAGE IMPLEMENTING GENETIC ALGORITHM}

A package has been developed using the genetic algorithm to automate the optimization of the designs of those collectors, which were earlier used to validate the random walk technique. This package has been tested in a similar way as the random walk package. For the symmetric collector, the population size, the chromosome length, the probability of crossover, and the maximum number of generations are taken to be $8,12,1$, and 50 , respectively; in the case of the asymmetric collector, these values are $8,6,1$, and 50 , respectively.

\section{A. Axisymmetric Collector}

After several trials, it was observed that in almost all the trials, the present package was capable of achieving an efficiency of more than $90 \%$ for the four-stage axisymmetric collector. In Fig. 10, the improvement in collector efficiency is plotted after different numbers of generations for four different trials to show the intermediate steps toward convergence. The optimization 


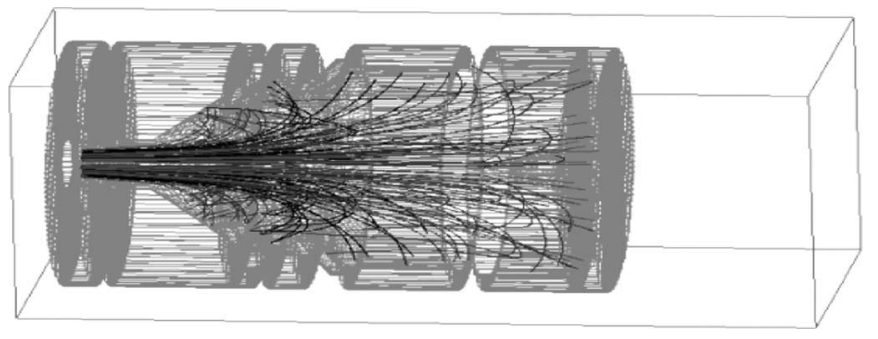

Fig. 11. Optimized four-stage axisymmetric collector with primary electron trajectories. Efficiency of the collector is nearly $91 \%$.

for all trials started from the same collector geometry, and the number of collectors simulated in each generation was the same as the population number. As the initial population in the genetic algorithm is created randomly and is nonrepetitive (each time the package is executed, the initial population is different), the intermediate steps are different, although the optimized results are nearly the same. For any trial, the amount of change in a specific dimension in any intermediate step is different. A maximum and a minimum limit have been set for the amount to be changed in any dimension in a single step. Any value between these limits can be chosen randomly to make a change in that dimension of the electrode.

The geometry of the four-stage collector with the primary trajectories after optimization is shown in Fig. 11. Different aspects of fabrication and practical issues of the collector, e.g., electrode thickness and gap between the electrodes, have been taken care of while optimizing its geometry (see Fig. 3). In the present example, the efficiency of the optimized collector is nearly $91 \%$, which is an improvement by more than 20 percentage points from the initial collector geometry. A careful look at the collector geometry in Fig. 5, which is optimized via the random walk algorithm, reveals that a few trajectories are hitting at the front face of electrode 3 and electrode 4, which could have traveled a longer path before they are collected. The energies of these electrons are not recovered fully, and this reduces the collector efficiency and may cause excessive heating. On the contrary, nearly all the primaries in Fig. 11 are collected on the backs of the electrodes. This will also help in recapturing the secondary electrons. Another comparison between the two figures shows that the length of the collector has been reduced by nearly $30 \%$, and this has reduced both its volume and its weight. This is important for spaceborne and airborne applications.

\section{B. Asymmetric Collector}

To further check the suitability of the genetic algorithm, the two-stage asymmetric collector was optimized. After a number of trials, it was observed that the optimized collector efficiency is $84 \%$ for nearly all the trials. The best collector efficiency at intermediate steps for four different trials is plotted in Fig. 12. As in the previous example, the starting geometry is the same in all four cases. The geometry of the two-stage asymmetric collector after optimization is shown in Fig. 13. The improvement in collector efficiency is about 5 percentage points.

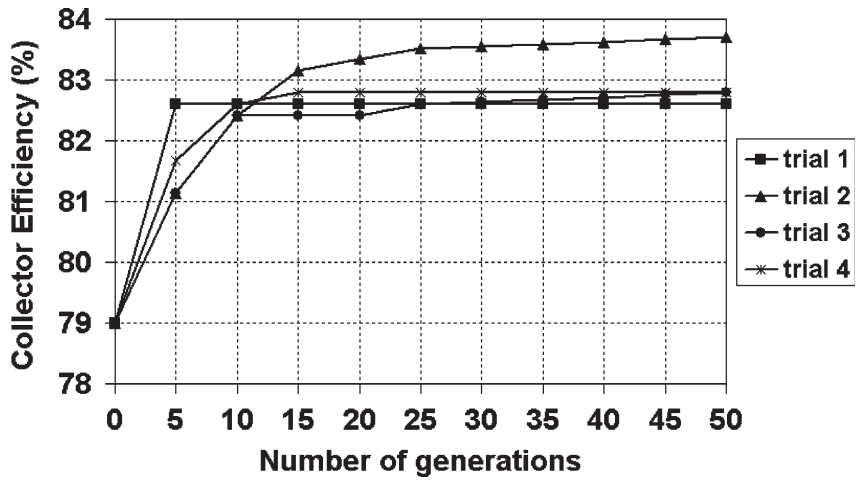

Fig. 12. Improvement in efficiency for a two-stage asymmetric collector versus the number of generations (the starting geometry is the same for all trials).

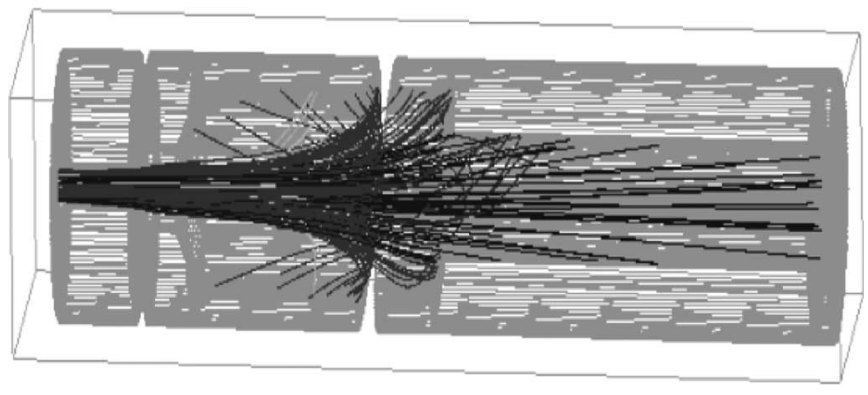

Fig. 13. Optimized two-stage asymmetric collector with primary electron trajectories. Efficiency of the collector is nearly $84 \%$.

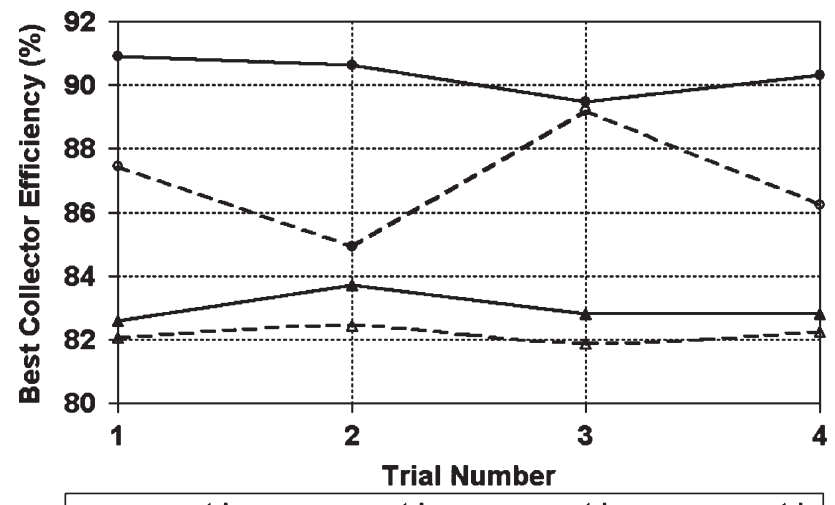

Fig. 14. Best collector efficiency for each trial showing the repeatability of the results. The curves with dotted lines are the random walk method, and the solid lines are the genetic algorithm.

\section{COMPARISON}

The performance of the random walk algorithm is compared with the genetic algorithm on the basis of the simulated results of both symmetric and asymmetric collectors. The advantages and disadvantages in terms of consistency, computational time, accuracy, and implementation are discussed in detail.

\section{A. Consistency}

The best collector efficiency achieved through the genetic algorithm and the random walk method for four different trials against the trial number is plotted in Fig. 14. In the case of the genetic algorithm, the best efficiency for both collectors 
remains nearly the same across all trials, whereas the random walk method is less consistent, although only the best four trials are considered.

\section{B. Computational Time}

The total number of simulations carried out for both collectors in each trial using the genetic algorithm is 400 (50 generations with eight collector simulations per generation). For the four-stage symmetric collector, the computational time for each trial is about $100 \mathrm{~h}$, whereas for the two-stage asymmetric collector, the computational time is about $60 \mathrm{~h}$. In the case of the random walk method, the times for each trial are 125 and $75 \mathrm{~h}$ for the four-stage symmetric and two-stage asymmetric collectors, respectively. In the latter case, only the best four trials are considered for comparison purposes. If, however, the total central processing unit time includes the time for all trials (not just the best four), then the average time for a successful trial using the random walk method will be much higher.

\section{Accuracy}

It is observed from Fig. 14 that the optimized performance of the best four trials using the random walk method is sometimes close to what is achieved using the genetic algorithm. More trials may be able to improve the performance of the collectors; however, this will increase the computational time.

\section{Implementation}

The advantage of the random walk method is its simplicity in implementation. However, there is always a possibility of the search being trapped in a local maximum due to the random nature of the algorithm. This can be overcome by changing both the starting point and the search path at different trials. In the example of the four-stage axisymmetric collector, the search is trapped in a local maximum after 100 iterations in trial 1 (see Table I). A better optimization is achieved by changing the path of the optimization in trial 3. Considering several other intermediate trials for which the method failed, it can be said that a large number of trials are necessary to achieve the optimized geometry.

On the contrary, the genetic algorithm employs a multidirectional search. It uses the geometry and performance information along with the search path from the chromosomes of each generation to create the population of a new generation through the natural evolution process. This makes the genetic algorithm more effective than the random walk method. For complex problems where both the number of variables and the search space are large, the genetic algorithm is the best choice if it is used efficiently. Previous experience in design and simulation of collectors may be helpful in implementing this algorithm for optimization purposes. Variations of this algorithm may be tried to reduce the time taken to solve complex problems. Each time it is executed, the genetic algorithm follows a different path toward the optimization, as the intermediate steps are different and the starting population is generated randomly, but the final outcome in each case is nearly the same. The repeatability feature of the genetic algorithm makes it a better choice over the random walk method.

\section{CONCLUSION}

Genetic algorithm and random walk methods have been implemented using a 3-D collector simulator for the optimization of both axisymmetric and asymmetric collectors. These techniques can be implemented using any similar suitable code. The optimized performances of the best four trials using the random walk algorithm have been presented. A large number of trials are required to achieve the optimized geometry using this algorithm. On the other hand, repeatability of the results was achieved in all the trials of the genetic algorithm. To reduce the total computation time, the optimization of both of these collectors has been carried out without including the secondary electrons. If the effect of secondaries is included, then the efficiency is expected to be reduced by a few percent depending on the material used for the collector electrodes.

\section{ACKNOWLEDGMENT}

The authors would like to thank Dr. W. L. Menninger, L-3 Communications Electron Technologies, Inc., for his valuable suggestions to improve this paper; INP-DME for granting permission to use KOBRA3 for this research; and the Head of the Department of Engineering, Lancaster University, for providing facilities to carry out this work. One author, T K. Ghosh, would like to thank the Director of the Central Electronics Engineering Research Institute, Pilani, India, for providing leave and support during the project and $\mathrm{e} 2 \mathrm{v}$ technologies, U.K., for their support during the preparation of this paper.

\section{REFERENCES}

[1] H. G. Kosmahl, A novel axi-symmetric electrostatic collector for linear beam microwave tubes, 1971. NASA Tech. Note TN D-6093.

[2] W. Neugebauer and T. Mihran, "A ten stage depressed collector for improving klystron efficiency,” IEEE Trans. Electron Devices, vol. ED-19, no. 1, pp. 111-121, Jan. 1972.

[3] H. G. Kosmahl, "Modern multistage depressed collectors-A review," IEEE Trans. Electron Devices, vol. ED-70, no. 11, pp. 1325-1334, Nov. 1982.

[4] A. C. Schram, "TWT efficiency improvement using multi-stage collectors," Microw. J., vol. 18, no. 5, pp. 31-33, Aug. 1975.

[5] R. Forman, "Secondary-electron-emission properties of conducting surfaces with application to multistage depressed collectors for microwave amplifiers," NASA, Washington, DC, NASA TP-1097, 1977.

[6] A. N. Curren and K. A. Jansen, "Secondary electron emission characteristics of ion-textured copper and high-purity isotropic graphite surfaces," NASA, Washington, DC, NASA TP-2342, 1984.

[7] A. N. Curren, "Carbon and carbon-coated electrodes for multistage depressed collectors for electron-beam devices-A technology review," IEEE Trans. Electron Devices, vol. ED-33, no. 11, pp. 1902-1913, Nov. 1986.

[8] P. Ramins and B. T. Ebihara, "Improvement in MDC and TWT overall efficiency through the application of carbon electrode surfaces," IEEE Trans. Electron Devices, vol. ED-33, no. 11, pp. 1915-1924, Nov. 1986.

[9] T. Okoshi, E. Chiu, and S. Matsuki, "The tilted electric field soft-landing collector and its application to a travelling-wave tube," IEEE Trans. Electron Devices, vol. ED-19, no. 1, pp. 104-110, Jan. 1972.

[10] J. R. Hechtel, "A novel electrostatic-focusing depressed collector for linear beam tubes," IEEE Trans. Electron Devices, vol. ED-24, no. 1, pp. 45-52, Jan. 1977.

[11] K. R. Vaden, J. D. Wilson, and B. A. Bulson, "A simulated annealing algorithm for the optimization of multistage depressed collector efficiency," in Proc. 3rd IEEE Int. Vac. Electron. Conf., Apr. 23-25, 2002, pp. 164-165. 
[12] T. K. Ghosh and R. G. Carter, "Design optimisation of multistage depressed collectors for high efficiency travelling wave tubes using genetic algorithm," in Proc. 3rd IEEE Int. Vac. Electron. Conf., Apr. 23-25, 2002, pp. $158-159$.

[13] T. K. Ghosh, "Three dimensional modeling and optimization of multistage depressed collectors," Ph.D. dissertation, Dept. Eng., Lancaster Univ., Lancaster, U.K., 2002.

[14] L. Kumar, P. Spadtke, R. G. Carter, and D. Perring, "Three-dimensional simulation of multistage depressed collectors on micro-computers," IEEE Trans. Electron Devices, vol. 42, no. 9, pp. 1663-1673, Sep. 1995.

[15] V. Srivastava and R. G. Carter, "Improved 2.5-dimensional large signal model for helix TWTs \& its application for stability analysis," Univ. Lancaster, Lancaster, U.K., Report MRG/98/1, Feb. 1998.

[16] M. N. Barber and B. W. Ninham, Random and Restricted Walks: Theory and Applications. New York: Gordon and Breach, Jun. 1970.

[17] F. Spitzer, Principles of Random Walk. New York: Springer-Verlag, Jan. 1976.

[18] Z. Michalewicz, Genetic Algorithm + Data Structure $=$ Evolution Programs. Berlin, Germany: Springer-Verlag, 1992, pp. 107-139.

[19] D. E. Goldberg, Genetic Algorithms in Search, Optimization, and Machine Learning. Reading, MA: Addison-Wesley, 1989, pp. 70-110.

[20] L. Davis, Handbook of Genetic Algorithms. New York: Van Nostrand, 1991, pp. 60-100.

[21] L. Davis, Genetic Algorithms and Simulated Annealing. London, U.K.: Pitman, 1990, pp. 111-136.

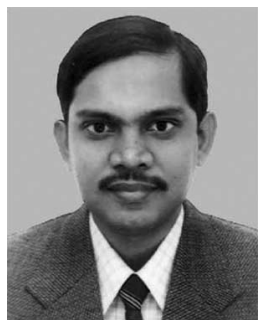

Tushar K. Ghosh (S'99-M'00) received the B.E. degree in electronics and telecommunication engineering from Bengal Engineering College, Howrah, India, in 1990, the M.E. degree in electronics and telecommunication engineering from Jadavpur University, Kolkata, India, in 1993, and the Ph.D. degree in engineering from Lancaster University, Lancaster, U.K., in 2002, for his work on 3-D modeling and optimization of multistage collectors.

From 1993 to 1994, he worked on electromagnetic pulse sensors as a Senior Research Fellow at Jadavpur University. In 1994, he joined the Central Electronics Engineering Research Institute (CEERI), Pilani, India, as a Project Scientist and was a Scientist from 1995 to 2004. During this period, he was a key member of the C-band and Ku-band space TWT project teams, where he was involved in the design and development activities. He has developed a number of computer codes implementing advanced optimization techniques, which are presently being used at CEERI for the design purpose. He joined e2v technologies, Chelmsford, U.K., in 2004 as a Principal Engineer, where he is currently involved in improving the design and modeling techniques of TWTs. He has authored or coauthored a number of papers published in international journals and conference proceedings. His primary interests are in vacuum device physics, modeling, and design.

Dr. Ghosh was awarded the Commonwealth Scholarship from 1999 to 2002. He was one of the first three recipients of the IEEE-EDS Graduate Student Fellowship Award in 2001.

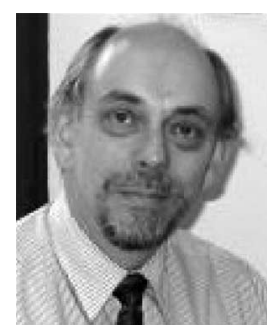

Richard G. Carter (M'97-SM'01) received the B.S. degree in physics from the University of Cambridge, Cambridge, U.K., in 1965 and the Ph.D. degree in electronic engineering from the University of Wales, Cardiff, U.K., in 1968, for his work on the propagation of waves on neutralized ion beams.

From 1968 to 1972, he worked on high-power traveling-wave tubes as a Development Engineer at English Electric Valve Co., Ltd. He joined the Department of Engineering, University of Lancaster, Lancaster, U.K., as a Lecturer in 1972 and was promoted to Senior Lecturer in 1986 and Professor of electronic engineering in 1996. His research interests include electromagnetics and microwave engineering, with particular reference to the theory, design, and computer modeling of microwave tubes and particle accelerators.

Prof. Carter is a Fellow of the Institution of Engineering and Technology and a member of the Vacuum Electronics and Compact Modeling Technical Committees of the IEEE Electron Devices Society. 